\title{
Repetitive transforaminal steroid injections in cervical radiculopathy: a prospective outcome study including 140 patients
}

Authors Liselotte Persson, Leif Anderberg

Institution Department of Clinical Science, Neurosurgery, Lund University Hospital, Lund, Sweden

\begin{tabular}{l|l}
\hline Final class of \\
evidence-treatment \\
Study design \\
\hline RCT \\
Cohort \\
Case control \\
Case series \\
\hline Methods \\
\hline Concealed allocation (RCT) \\
\hline Intention to treat (RCT) \\
\hline Blinded/independent \\
evaluation of primary outcome
\end{tabular}

The definiton of the different classes of evidence is available on page 63.

\section{ABSTRACT}

Study design: Prospective case series.

Objective: To evaluate the effect of three repetitive transforaminal steroid injections in a large series of selected patients with cervical radiculopathy caused by spondylosis.

Methods: Consecutively, 140 patients with long-lasting medical history, clinical findings, and MRI indicating a cervical nerve root origin based on degenerative disease and a positive selective transforaminal diagnostic nerve root blocks with local anesthetics resulting in at least 50\% temporary arm pain reduction were included.

Before treatment started, patients underwent a clinical examination by a neurosurgeon. All patients were followed-up and evaluated by one physiotherapist at the neurosurgery outpatient clinic. A designed outcome questionnaire including Neck Disability Index (NDI), Symptoms Frequency Index, and Visual Analog Scale for pain intensity were used.

A series of three transforaminal steroid injections, with 3 weeks in between, were performed by a neuroradiologist using image intensifier guidance in an $\mathrm{x}$-ray suite. At 12-14 weeks after the first injection, follow-up was performed. Criteria for positive response to the treatment was $>50 \%$ radicular arm pain reduction. Except for occasional painkillers, no other treatment was given to the patients.

Results: Positive response to the treatment was achieved in $49 \%(n=69)$ with a significant difference in NDI and pain intensity between responders and nonresponders.

Conclusions: Repetitive transforaminal steroid injections may reduce symptoms (frequency, intensity, and fewer limitations of daily living activities) of radiculopathy in patients with degenerative disease in the cervical spine at a short time follow-up. 


\section{STUDY RATIONALE AND CONTEXT}

Treatment strategies for cervical radiculopathy caused by disc degeneration and spondylosis include ergonomic adjustments, analgesic medications including opioid and nonsteroidal antiinflammatories, physiotherapy, temporary immobilization with a collar, and, in case of failure of conservative treatments, eventually surgery $[1,2]$. Transforaminal steroid injections have sporadically been reported in the literature as an alternative for treatment of cervical radiculopathy [3-10]. The use of such injections is controversial and no studies with strong evidence for their effectiveness have been published. However, severe complications have been reported in a small number of patients following transforaminal steroid injections [11-13]. We have previously reported on favorable results using transforaminal steroid injections for many years as an alternative treatment to surgery in select patients [3]. We have also noted that patients often needed repetitive injections to achieve satisfactory results. The need for repetitive injections has been reported in the literature [9].

\section{OBJECTIVE}

To evaluate effects of transforaminal steroid injections in a series of three injections, within 3-week intervals, in patients with well-defined radiculopathy caused by spondylosis in the cervical spine.

\section{METHODS}

Study design: Prospective case series.

\section{Inclusion criteria:}

- Consecutively referred patients with long-lasting medical history and clinical findings indicating a cervical nerve root origin, with pain radiating from the neck into the arm.

- Presence of significant degenerative pathology of the cervical spine at one or two levels on the same side as the radicular pain confirmed by magnetic resonance imaging (MRI) strongly suggesting nerve root compression due to spondylosis/foraminal stenosis as cause of the symptoms.

- Patients had undergone a diagnostic selective transforaminal nerve root block (SNRB) with positive response to one or two nerve roots, indicating a pain reduction $>50 \%$.

Exclusion criteria: Patients older than 75 years, patients with communication problems (Fig $\mathbf{1}$ ).

Patient population: During 3 years 140 of 150 consecutive patients were enrolled. There were 78 women $(56 \%)$ and 62 men (44\%) (Table 1). The mean age was 52 $( \pm 8.2)$ years and the mean symptom duration was 23 $( \pm 16)$ months. Twenty-four patients $(17 \%)$ underwent previous surgery for similar symptoms and were now readmitted because of new symptoms.

Study design: All patients had a clinical examination by a senior neurosurgeon and received an MRI scan of the cervical spine. To further sharpen the diagnosis, confirming the radicular arm pain had nerve root origin, all patients had undergone a diagnostic nerve root block with local anesthetics (SNRB) performed on the nerve root/roots presenting with significant degenerative MRI pathology [3]. A response was rated as positive if the pain intensity in the arm was reduced $50 \%$ or more. Only nerve roots with positive response to the diagnostic block received steroid treatment [5].

- A series of three transforaminal steroid injections was given within 3 weeks between injections. Except from occasional analgesics, the study treatment protocol included steroid injections as only treatment (Fig 1).

- Before treatment and 14 weeks after the first injection, all patients were examined by a senior neurosurgeon and by a specially trained physiotherapist at the outpatient clinic, according to a set protocol. The following variables were recorded: pain history, 
pain distribution, and pain intensity in the neckshoulder-arm, occurrence of headache, muscle tenderness in the neck, paresthesia, and sensory loss. The patients were also asked to complete two self-administered questionnaires-Neck Disability Index (NDI) and Symptoms Frequency Index (SFI). Before each steroid injection, pain intensity in the neck-shoulder-arm were evaluated using Visual Analog Scale (VAS). An outcome evaluation was performed 14 weeks after the first injection at the outpatient clinic by the same physiotherapist and a neurosurgeon. Before the follow-up appointment, the patients had been sent questionnaires to complete and bring to the outpatient clinic (pain drawing, VAS, NDI, SFI, and an outcome scale) (Fig 1).

Fig 1 Study design.

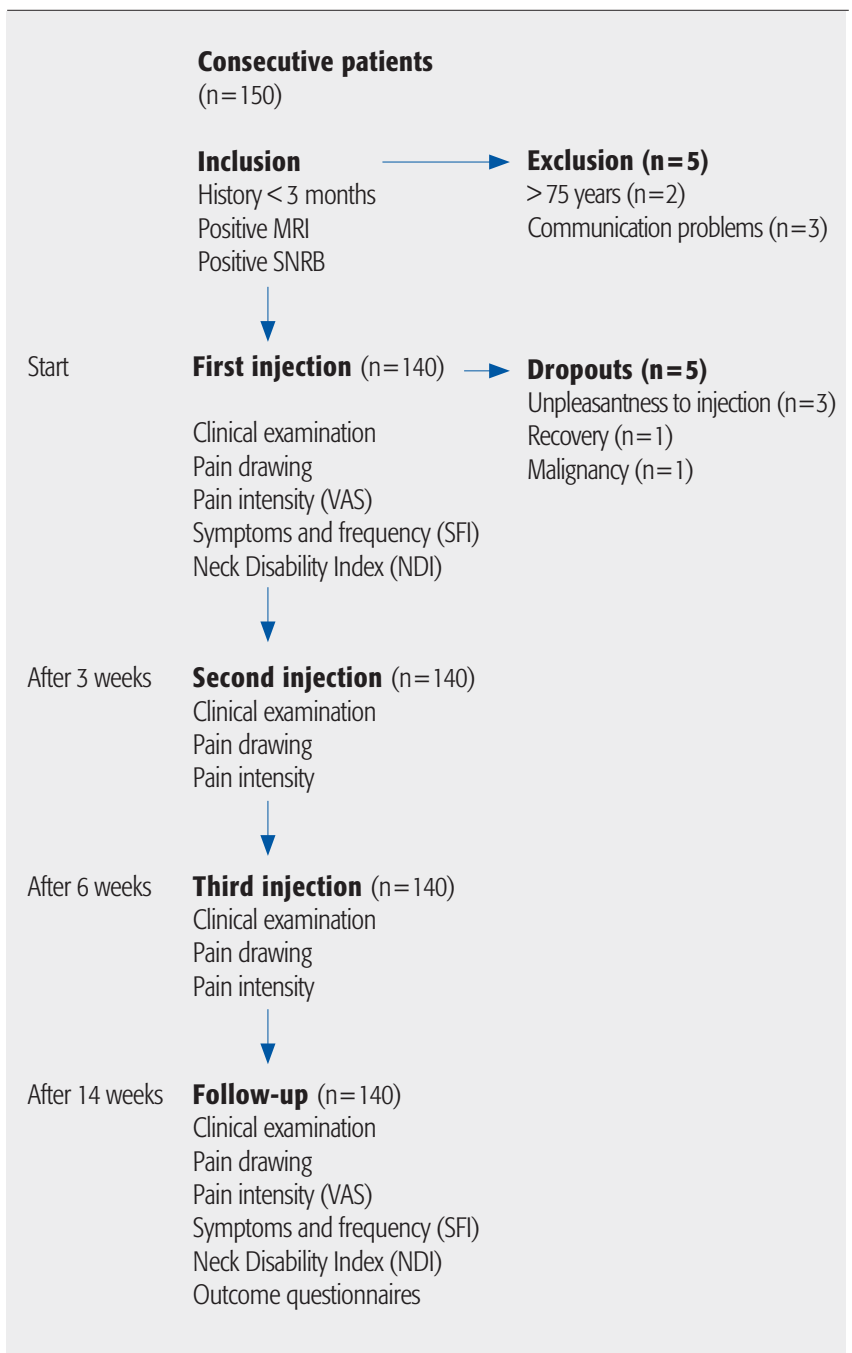

\begin{tabular}{lll}
\hline Table 1 & Selected demographic and social data.* \\
\hline No. (\%) & Women & $78(56)$ \\
\hline & Men & $62(44)$ \\
\hline Age, y & Mean \pm SD & $52 \pm 8.9$ \\
\hline & Median (ql-q3) & $52(46-58)$ \\
\hline Pain duration, mo & Mean \pm SD & $23 \pm 16$ \\
\hline & Median (q1-q3) & $20(12-30)$ \\
\hline Symptomatic nerve root, No. (\%) & CIV & $5(4)$ \\
\hline & CV & $9(6)$ \\
\hline & CVI & $72(52)$ \\
\hline & CVII & $45(32)$ \\
\hline & CVIII & $9(6)$ \\
\hline Symptomatic side, No. (\%) & Right & $75(54)$ \\
\hline & Left & $65(46)$ \\
\hline
\end{tabular}

\section{ASSESSMENTS}

- Pain distribution and pain intensity: A drawing was used to describe the location and quality of pain. The mean pain intensity experienced the last $1-2$ days in the neck-shoulder-arm was noted on three different VAS graded from 0 to 100 .

- Limitations of daily living: NDI is a questionnaire to measure limitation in daily living due to the neckshoulder-arm pain. The 10 items in NDI are scored 0 (no activity limitation) to 5 (major activity limitations), with a maximum total score of 50 . To get a percentage score, the patient's degree of disability, the scores on each question were added, and the total score was divided by the maximum score 50 , transformed to percentage score $\times 100$ (percentage points).

- Frequency of symptoms: SFI is a questionnaire for patients to report their frequency of symptoms on a scale of 1 to 5 (never, seldom, sometimes, daily, and always). The items include pain in the neck-shoulderarm, neck stiffness, headache, numbness in the hands, hand-weakness, irritation, lack of concentration, sleep disturbances, unsteadiness, and pain radiating to the chest region.

- Outcome questionnaire: This questionnaire is for the patient's subjective changes in symptoms associated with the radicular pain (pain in the neck-shoulder-arm, concentration, irritation, headache, neck-shoulder-arm movements) after steroid injections. Patients could choose among unchanged, better, and worse [7].

* Mean \pm SD and median, quartiles (q1 and q3) are given. $\mathrm{N}=140$. 


\section{INJECTION TECHNIQUE}

- The same technique was used for the diagnostic as well as for the treatment injections. Using a lateral approach a needle was introduced into the neuroforamen with the aid of image intensifier guidance performed in an $\mathrm{x}$-ray suite by a neuroradiologist as described in earlier publications from our group [3]. The needle position was confirmed with the use of contrast media (0.1-0.3 mL). The effect of the temporary nerve root block concerning the pain in the neck, shoulder, and arm was then assessed with VAS, immediately before and 30 minutes after the diagnostic block by a physiotherapist at the outpatient clinic (Fig 1).

\section{ANALYSIS OF DATA}

- The percentage relief of pain at follow-up was calculated for each patient as a difference between before treatment and at follow-up. Nonparametric tests were used. For the group comparison, before and after steroid injections, the Wilcoxon matched-pairs signed rank test was used. For comparison between patients with and without positive response ( $\geq 50 \%$ pain reduction) and better outcome, comparison with Mann-Whitney U test was used. Correlation between variables was analyzed with Spearman rank correlation coefficients. A difference of $P<.05$ was considered statistically significant.

Fig 2 Pain distribution according to VAS for all patients $(n=140)$ during the study.

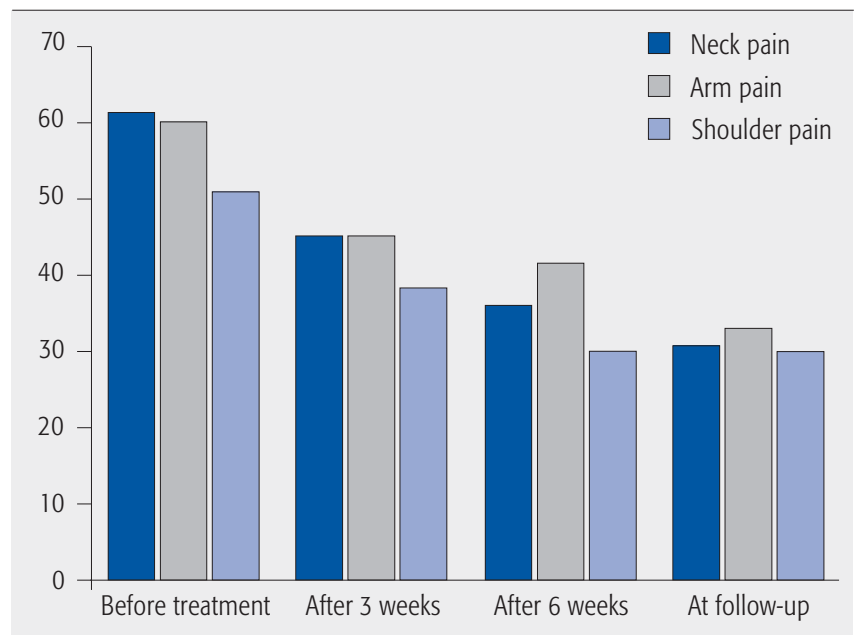

\section{ETHICS}

- The Regional Ethical Review board approved the study.

\section{RESULTS}

- Pain intensity: For all patients the pain intensity in the neck, shoulder, and the affected arm was statistically significantly $(P<.001)$ lower at the final outcomes evaluation $(n=140)$. The median pain intensity for all parameters was reduced about $50 \%$ according to VAS. The pain intensity tended to decrease with each injection in all parameters (Fig 2).

- Response to treatment: At follow-up 69 (49\%) of the 140 patients reported $\geq 50 \%$ of arm pain reduction on VAS, and were rated as having a positive response to the steroid injections. Of these 69 patients, 38 (27\%) had absolutely no arm pain at follow up (Table 2). At start there was no significant difference in pain intensity in the neck, shoulder, and the affected arm among responders $(n=69)$ and nonresponders $(n=71)$. At follow-up the difference between the two groups was statistically significant $(P<.001)$.

- Symptoms frequency index: Before treatment, $91 \%$ of patients $(n=127)$ reported daily or constantly neckshoulder-arm pain. Neck stiffness and numbness in the hand were also daily or constantly occurring symptoms.

Table 2 Localizations of pain reduction at follow-up in patients with positive response to treatment $(n=69)$.*

\begin{tabular}{lll}
\hline Pain & $\begin{array}{l}\text { No. (\%) with } \geq \mathbf{5 0 \%} \\
\text { pain reduction on VAS }\end{array}$ & $\begin{array}{l}\text { No. (\%) with } \mathbf{1 0 0 \%} \\
\text { pain reduction on VAS }\end{array}$ \\
\hline Neck & $68(49)$ & $28(20)$ \\
\hline Shoulder & $65(46)$ & $34(24)$ \\
\hline Arm & $69(49)$ & $38(27)$ \\
\hline
\end{tabular}

* VAS indicates Visual Analog Scale. 
Other frequently occurring symptoms were headache, described often or constantly by $54 \%$ of patients, and $55 \%$ reported sleeping problems caused by the neckshoulder-arm pain. Cognitive and other secondary symptoms due to the cervical radiculopathy, such as irritation, feeling of anxiety, problems with memory, and ability to concentrate were also frequently reported. At follow up, the frequency of symptoms for all patients $(n=140)$ was generally reduced except pain radiating to the chest, storage of memory, dizziness, and feeling of anxiety. Sixty-five $(46 \%)$ of the patients reported less frequency of pain, $46 \%$ reported less frequency of headache, and $45 \%$ reported less frequency of sleeping problems.

- Limitation of daily living (NDI): The NDI percentage score of the patient's degree of disability on each question are present in Table 3. Four patients did not fulfill the final NDI score at follow-up. There was no significant gender difference on the NDI score before $(P=.13)$ or after $(P=.06)$ the steroid injections series. Pain intensity, headache, and sleep improved the most. Of the 136 patients who had completed the final follow-up questionnaire, 91 (67\%) had individually improved on the NDI score. For patients with positive response to the treatment, there was a significant difference in the NDI outcome score compared with nonresponders $(P=.0001)$. The mean NDI in patients with positive response to the treatment was reduced by approximately $30 \%$ (Table 3).

Table 3 Neck Disability Index (NDI) total score in percentage before and at follow-up in the group with positive response and in nonresponders to the treatment.

\begin{tabular}{llll}
\hline & $\begin{array}{l}\text { NDI before } \\
\text { treatment }\end{array}$ & $\begin{array}{l}\text { NDI at } \\
\text { follow-up }\end{array}$ & $\boldsymbol{P}$ \\
\hline Positive response $(\mathbf{n = 6 9 )}$ & & & \\
\hline Mean (median) & $44(40)$ & $30(28)$ & .000 \\
\hline SD (range) & $\pm 18.0(6-94)$ & $\pm 18.3(4-86)$ & \\
\hline Nonresponders (n=71) & & & \\
\hline Mean (median) & $47(46)$ & $46(48)$ & .698 \\
\hline SD (range) & $\pm 14.1(14-76)$ & $\pm 13.5(10-76)$ & \\
\hline
\end{tabular}

- Quality of life outcome questionnaires: Of 140 patients, $109(78 \%)$ reported improvement in one or several symptoms. Neck, shoulder, and arm pain were the symptoms that had the best response to the treatment. Headaches improved in $44 \%$ of the patients and $46 \%$ reported a decreased intake of analgesics. Shoulder mobility, sensibility, arm, and hand power showed the least improvement (Table 4). Patients who reported improvement in the outcome questionnaire showed a significantly $(P=.003)$ reduced NDI score. These patients had also significantly less pain intensity in the neck-shoulder-arm $(P<.001)$ and headache intensity $(P<.025)$ according to VAS. There was no significant difference in the NDI score between patients with positive responds to the treatment and those who reported improvement in the outcome questionnaire $(P>.05)$.

- Complications: Three patients reported unpleasantness and increased pain in the arm/neck after the first steroid injections within 1-2 days. None of the patients experienced any moderate or severe complication and all could receive treatment on an outpatient basis.
Table 4 Patients' subjective effects and reactions at follow-up $(n=140)$.

\begin{tabular}{llll}
\hline & Unchanged & Better & Worse \\
\hline $\begin{array}{l}\text { How is your overall } \\
\text { reaction to the treatment? }\end{array}$ & $28(20 \%)$ & $109(78 \%)$ & $3(2 \%)$ \\
\hline $\begin{array}{l}\text { Have you felt any changes } \\
\text { in neck pain? }\end{array}$ & $54(39 \%)$ & $81(58 \%)$ & $5(3 \%)$ \\
\hline $\begin{array}{l}\text { Have you felt any changes } \\
\text { in arm pain? }\end{array}$ & $41(29 \%)$ & $94(67 \%)$ & $5(4 \%)$ \\
\hline $\begin{array}{l}\text { Have you felt any changes } \\
\text { in headache? }\end{array}$ & $72(52 \%)$ & $62(44 \%)$ & $6(4 \%)$ \\
\hline $\begin{array}{l}\text { Has your neck mobility } \\
\text { undergone any changes? }\end{array}$ & $95(68 \%)$ & $43(31 \%)$ & $2(1 \%)$ \\
\hline $\begin{array}{l}\text { Have your shoulder/arm } \\
\text { mobility undergone any } \\
\text { changes? }\end{array}$ & $99(71 \%)$ & $41(29 \%)$ & $0(0 \%)$ \\
\hline $\begin{array}{l}\text { Have you changed your } \\
\text { intake of analgesics? }\end{array}$ & $71(51 \%)$ & $65(46 \%)$ & $4(3 \%)$ \\
\hline $\begin{array}{l}\text { Has your quality of sleep } \\
\text { undergone any changes? }\end{array}$ & $82(59 \%)$ & $51(36 \%)$ & $7(5 \%)$ \\
\hline
\end{tabular}




\section{DISCUSSION}

- Of 140 patients treated for cervical spondylotic radiculopathy, with a total of 420 transforaminal steroid injections, we report positive response to the treatment in $49 \%$ (defined as $\geq 50 \%$ radicular pain reduction). The strategy of using a series of treatment injections appeared successful as our continuous follow-up revealed a trend toward pain decrease with every injection. This improvement was consistent with our other results throughout the follow-up protocol. The patients with positive response to the treatment moved from severe to moderate disability according to the NDI. However, symptoms from spondylotic radiculopathy often decrease without invasive treatment in many patients over months to years [2]. As all patients before inclusion had undergone at least 6 months of conservative treatment without success, our results suggest a clinical treatment effect.

- No moderate or severe complications were seen and this is related to the use of a standardized injection technique performed by experienced neuroradiologists. Based on our experience this technique is safe with experienced radiologists.

- Strengths: This study used clear indications to confirm the diagnosis, with both clinical and imaging concurrence required. This study was prospective, with an adequate number of enrolled patients, and with an unbiased observer performing a standardized followup. This study evaluated parameters as presence of symptoms, frequency, pain intensity, and distribution as well as secondary symptoms as a result of the pain and the impact on daily living.

- Limitations: The weakness in this study consists of a lack of a control cohort. We were not able to differentiate the potential influence of the natural course of the disease or possible placebo effects from the effect of injections. Another limitation is the short follow-up of the observation time. A longer follow-up might reveal a need for further treatment in those who responded to the treatment. A randomized study including other treatments, for example surgery, with long enough follow-up, could detect if there were an indication for using transforaminal steroid injections in the treatment of cervical spondylotic radiculopathy. However, the results in this study indicate that the use of such treatment might help a number of patients to move from severe to moderate disability status; thereby, nonsurgical management could help them through the most painful part of the natural history of the disease.
- Further studies on long-term effect from steroid injections in cervical radiculopathy are necessary. It is also important to try to identify the characteristics of individual patients who are more likely to respond to this form of intervention and differentiate these findings from patients without response to help in making a decision-making tree.

\section{CONCLUSIONS}

- Repetitive transforaminal steroid injections may have a positive short-term effect on radicular pain, when using $50 \%$ reduction of radicular arm pain on VAS as cutoff for treatment effect.

- In experienced hands, using a standardized technique, the injections can be performed without serious complications.

- Further studies on long-term effects are necessary before the treatment can be generally recommended.

\section{ACKNOWLEDGMENT}

- We thank the staff at the Department of Neuroradiology, University of Lund, Sweden, for their support and cooperation and our statistics expert Håkan Löfquist for his help. 


\section{REFERENCES}

1. Carette S, Fehlings MG (2005) Clinical practice: cervical radiculopathy. $N$ Engl J Med; 353(4):392-399.

2. Persson LC, Carlsson CA, Carlsson JY (1997) Long-lasting cervical radicular pain managed with surgery, physiotherapy, or a cervical collar: a prospective, randomized study. Spine (Phila Pa 1976); 22(7):751-758.

3. Anderberg $L$, Annertz $M$, Brandt $L$, et al (2004) Selective diagnostic nerve root blocks: correlation with clinical symptoms and MRIpathology. Acta Neurochir; 146(6):559-565.

4. Cyteval C, Fescquet N, Thomas E, et al (2004) Cervical radiculopathy: open study on percutaneous periradicularforaminal steroid infiltration performed under CT control in $30 \mathrm{pa}$ tients. AJNR Am J Neuroradiol; 25(3):441-445.

5. Anderberg L, Annertz M, Rydholm U, et al (2006) Selective diagnostic nerve root block for the evaluation of radicular pain in the multilevel degenerated cervical spine. Eur Spine J; 15(6):794-801.

6. Slipman CW, Chow DW (2002) Therapeutic spinal corticosteroid injections for the management of radiculopathies. Phys Med Rehabil Clin N Am; 13(3):697-711.

7. Anderberg $L$, Annertz $\mathbf{M}$, Persson $L$, et al (2007) Transforaminal steroid injections for the treatment of cervical radiculopathy: a prospective and randomised study. Eur Spine J; 16(3):321-328.

8. Kolstad F, Leivseth G, Nygaard OP (2005) Transforaminal steroid injections in the treatment of cervical radiculopathy: a prospective outcome study. Acta Neurochir (Wien); 147(10):1065-1070.

9. Bush K, Hillier S (1996) Outcome of cervical radiculopathy treated with periradicular/epidural corticosteroid injections: a prospective study with independent clinical review. Eur Spine J; 5(5):319-325.

10. Wolter T, Knoeller S, Berlis A, et al (2010) CTguided cervical selective nerve root block with a dorsal approach. AJNR Am J Neuroradiol; 31(10):1831-1836.

11. Furman MB, Giovanniello MT, O'Brien EM (2003) Incidence of intravascular penetration in transforaminal cervical epidural steroid injections. Spine; 28(1):21-25.

12. Huntoon MA, Martin DP (2004) Paralysis after transforaminal epidural injection and previous spinal surgery. Reg Anesth Pain Med; 29(5):494-495.

13. Ludwig MA, Burns SP (2005) Spinal cord infarction following cervical transforaminal epidural injection: a case report. Spine (Phila Pa 1976); 30(10):E266-268. 


\section{EDITORIAL PERSPECTIVE}

Our reviewers were split in their reviews. After a number of corrections by the authors, in the end the size of the cohort and the quality of data gathering with sound general methodology were decisive in favor of publication of this article.

As the authors point out it would have been helpful to create a control group - especially a nonoperative one without any form of intervention. Patients basically served as their own internal controls. The cumulative improvement of patients as shown in Fig 2 is impressive, assuming that all these patients had static symptoms for a more than a few weeks before receiving their injection treatment. The differentiation of 'responders' and 'nonresponders' as demonstrated in Table 4 clearly should be viewed with suspicion as by default the nonresponders were treatment failures and a statistical separation by virtue of the apportioning of 'responders' by their inclusion criteria will lead to a statistically significant difference, even if 'arm pain' was used as a primary selection point for the two groups.

From an outcome perspective two important aspects of nonoperative treatment are duration of symptom relief and return to regular function.

For an evaluation of duration of symptom relief, a 2-year followup would seem important; hopefully Anderberg and Persson will make arrangements to keep track of their cohort. This is notoriously difficult in patients treated nonoperatively. Perhaps the more regimented government-sponsored healthcare system in Sweden, the origin of this study, allows for longer tracking of even nonoperative patients.
The functional recovery question in nonoperatively treated patients may be affected by a 'subconscious' down regulation of activities on the part of affected individuals due to fear of recurrent symptoms. While the Neck Disability Index (NDI) tries to assess functional performance with its questions 3 (lifting), 4 (work), 8 (driving), and 10 (recreation), the actual functional performance of patients may remain decreased in those with ongoing symptoms because of the magnitude of functional inhibition being inadequately expressed in the NDI score due to an adaptive effect experience by the affected patients. In addition to providing differentiated results scales-as done with SF36 objective measures - such as strength or dexterity testing, quantified analgesics usage, or return to work may offer greater differentiation of the functional capacity of patients.

Finally, the authors accumulated an impressive data set and showed that a technically well-performed series of transforaminal cervical steroid injections can provide meaningful symptom relief in about half of patients with cervical radiculopathy. The challenge to Anderberg and Persson is now to identify the characteristics of the responders by clinical and/or radiographic parameters to help us guide such patients toward early interventional nonoperative care while the search for best possible care for the other half must go on. 